\title{
Atomic properties of Cd-like and Sn-like ions for the development of frequency standards and search for the variation of the fine-structure constant
}

\author{
M. S. Safronova ${ }^{1,2}$, V. A. Dzuba ${ }^{3}$, V. V. Flambaum ${ }^{3}$, U. I. Safronova ${ }^{4,5}$, S. G. Porsev ${ }^{1,6}$, and M. G. Kozlov ${ }^{6,7}$ \\ ${ }^{1}$ University of Delaware, Newark, Delaware, USA \\ ${ }^{2}$ Joint Quantum Institute, NIST and the University of Maryland, College Park, Maryland, USA \\ ${ }^{3}$ The University of New South Wales, Sydney, Australia \\ ${ }^{4}$ University of Nevada, Reno, Nevada, USA \\ ${ }^{5}$ University of Notre Dame, Notre Dame, Indiana, USA \\ ${ }^{6}$ Petersburg Nuclear Physics Institute, Gatchina, Russia and \\ ${ }^{7}$ St. Petersburg Electrotechnical University "LETI", St. Petersburg, Russia
}

(Dated: May 20, 2018)

\begin{abstract}
A high-precision relativistic calculations of Cd-like $\mathrm{Nd}^{12+}, \mathrm{Sm}^{14+}$ and Sn-like $\mathrm{Pr}^{9+}, \mathrm{Nd}^{10+}$ atomic properties is carried out using an approach that combines configuration interaction and a linearized coupled-cluster method. These ions have long-lived metastable states with transitions accessible by laser excitations, relatively simple electronic structure, high sensitivity to $\alpha$ variation, and stable isotopes. Breit and QED corrections were included into the calculations. Energies, transition wavelengths, electric- and magnetic-multipole reduced matrix elements, lifetimes, and sensitivity coefficients $q$ and $K$ to the variation of the fine-structure constant $\alpha$ were obtained. A detailed study of uncertainties was performed. Energies for similar Cd-like $\mathrm{Ba}^{8+}, \mathrm{La}^{9+}, \mathrm{Ce}^{10+}, \mathrm{Pr}^{11+}$ and Sn-like $\mathrm{Ba}^{6+}$ ions were calculated and compared with experiment for further tests of the accuracy.
\end{abstract}

\section{INTRODUCTION}

Last five years marked extraordinary improvements in both the accuracy and stability of optical frequency standards [1 3] . The most accurate trapped-ion clock based on quantum logic spectroscopy of an $\mathrm{Al}^{+}$ion was demonstrated in 2010 [1]. The fractional frequency uncertainty of $8.6 \times 10^{-18}$ was reported. The optical frequency standard based on ${ }^{88} \mathrm{Sr}^{+}$trapped ion with the total fractional frequency uncertainty of $2.3 \times 10^{-17}$ was reported in [4]. In 2013 , Yb lattice clock with instability of $8.6 \times 10^{-18}$ after only 7 hours of averaging [2] was reported. The $6.4 \times 10^{-18}$ accuracy was achieved with the Sr optical lattice clock [3] which represents a factor of 22 improvement in comparison with the best previous optical lattice clock. Cryogenic Sr optical lattice clocks with a relative frequency difference of $10^{-18}$ was demonstrated in [5].

Further development of even more precise frequency standards is essential for new tests of fundamental physics, search for the variation of fundamental constants, and very-long-baseline interferometry for telescope array synchronization. The most precise laboratory test of variation of the fine-structure constant $\alpha$ has been carried out by measuring the frequency ratio of $\mathrm{Al}^{+}$and $\mathrm{Hg}^{+}$optical atomic clocks with a fractional uncertainty of $5.2 \times 10^{-17}[\underline{6}]$. Furthermore, more precise clocks will enable the development of extremely sensitive quantum-based tools for geodesy, hydrology, climate change studies, inertial navigation, and tracking of deepspace probes [2, 3].

This remarkable progress poses the question of what are the novel schemes for the clock development that may achieve the accuracy at the next decimal point, $10^{-19}$. We can single out two types of new clock scheme proposals at the present time. The first set of proposals are for the development of a nuclear clock [7] based on the ${ }^{229} \mathrm{Th}$ nuclear transition that has an unusually low first excitation energy of only several $\mathrm{eV}$ making it accessible with laser excitation. The second set of proposals involves various transitions in highly-charged ions (HCI) [8-10]. The estimates of potential accuracy of clocks based on highlycharged ions and the ${ }^{229} \mathrm{Th}$ nuclear transition are similar, but most HCI clock proposals do not have a complication of dealing with the radioactive isotope.

Recent studies of uncertainties 9, 10] have shown that the fractional uncertainty of the transition frequency in the clocks based on HCIs can be smaller than $10^{-19}$. Estimated sensitivity to the variation of $\alpha$ for highly-charged ions approaches $10^{-20}$ per year [10], which may allow for tests of spatial variation of the fine-structure constant that may be indicated by the observational studies [11].

While HCIs lack strong electric-dipole transitions for laser cooling, some have strong M1 transitions. Moreover, sympathetic cooling may be employed similar to the scheme used in $\mathrm{Al}^{+}$clock, which is cooled using lasercooled $\mathrm{Be}^{+}$or $\mathrm{Mg}^{+}$ions [1]. The experimental investigations toward the sympathetic cooling of HCIs and the precision laser spectroscopy of forbidden transitions are in progress [12 15]. A cooling scheme combining laser cooling of $\mathrm{Be}^{+}$ions and sympathetic cooling of $\mathrm{Xe}^{44+}$ by Coulomb collisions with the cold $\mathrm{Be}^{+}$ions has been demonstrated in [16]. In 2011, the evaporative cooling of $\mathrm{Ar}^{16+}$ in a Penning trap was demonstrated [12]. A novel extraction technique based on the excitation of a coherent axial oscillation which allowed to monitor the cooling process and to extract HCI bunches of high density and low momentum spread was also demonstrated [12]. Laser cooling of $\mathrm{Mg}^{+}$ions in a Penning trap for sympathetic cooling of highly-charged ions was demonstrated in [13]. Storage and cooling of highly-charged ions require ultrahigh vacuum levels. These can be obtained by cryogenic methods, and a linear Paul trap operating at $4 \mathrm{~K}$ capable 
of very long ion storage times of about $30 \mathrm{~h}$ was recently developed in 14, 15]. Capture and isolation of highlycharged ions in a unitary Penning trap extracted from an electron beam ion trap (EBIT) at NIST was demonstrated in [17]. The observed energy distribution was 60 times smaller than typically expected for ions inside an EBIT without applying any active cooling [17].

In a recent work, we proposed 10 highly-charged ions that belong to Ag-like, In-like, Cd-like, and Sn-like isoelectronic sequences as candidates for the development of next generation atomic clocks, search for variation of fine-structure constant, and quantum information [18]. Ag-like and In-like highly-charged ions have been further considered in Ref. [19].

In this work, we carried out detailed high-precision study of Cd-like $\mathrm{Nd}^{12+}, \mathrm{Sm}^{14+}$ and Sn-like $\mathrm{Pr}^{9+}$, $\mathrm{Nd}^{10+}$ highly-charged ions using an approach that combines configuration interaction $(\mathrm{CI})$ and a variant of the coupled-cluster method. Breit and quantum electrodynamic (QED) corrections were included into the calculations. Our calculations include energies, transition wavelengths, electric-dipole, electric-quadrupole, electric-octupole, magnetic-dipole, magnetic-quadrupole, magnetic-octupole reduced matrix elements, lifetimes, and sensitivity coefficients to $\alpha$-variation $q$ and $K$. We carried out extensive study of the uncertainties of our results. Two types of calculations were carried out for Sn-like ions, treating these ions as systems with two and four valence electrons to ensure that all important configurations were taken into account. Energies for similar Cd-like $\mathrm{Ba}^{8+}, \mathrm{La}^{9+}, \mathrm{Ce}^{10+}, \mathrm{Pr}^{11+}$ and Sn-like $\mathrm{Ba}^{6+}$ ions, where the experimental values are available, were calculated and compared with experiment for further tests of accuracy. Our values are in excellent agreement with experimental energies from 2022 for Cd-like $\mathrm{Ba}^{8+}$, $\mathrm{La}^{9+}, \mathrm{Ce}^{10+}$, with similar level of agreement for all three ions. However, we find a significant discrepancy with experimental values from [23, 24] for $\mathrm{Pr}^{11+}$ and $\mathrm{Nd}^{+12}$ which might indicate a problem with the experimental level identification. Detailed study of higher-order, Breit, QED, and higher partial wave contributions was carried out to evaluate uncertainties of the final results for each ion.

We start with the brief description of the CI+all-order method used in this work in Section [II. The results for Cd-like and Sn-like ions are presented in Sections III] and [IV] respectively.

\section{METHOD}

The Cd-like ions are divalent systems with two valence electrons above the $1 s^{2} 2 s^{2} 2 p^{6} 3 s^{2} 3 p^{6} 3 d^{10} 4 s^{2} 4 p^{6} 4 d^{10}$ core. We use a CI+all-order method developed in [25, 26] that combines the modified linearized single-double coupledcluster approach with configuration interaction. The CI many-electron wave function is obtained as a linear combination of all distinct states of a given angular momen- tum $J$ and parity [27]:

$$
\Psi_{J}=\sum_{i} c_{i} \Phi_{i} .
$$

The CI + many-body perturbation theory (MBPT) approach developed in [28] allows one to incorporate core excitations in the CI method by including perturbation theory terms into an effective Hamiltonian $H^{\text {eff }}$. The one-body part $H_{1}$ is modified to include the correlation potential $\Sigma_{1}$ that accounts for one-body part of the corevalence correlations:

$$
H_{1} \rightarrow H_{1}+\Sigma_{1}
$$

and the two-body Coulomb interaction term $\mathrm{H}_{2}$ is modified by including the two-body part of core-valence interaction that represents screening of the Coulomb interaction by valence electrons;

$$
H_{2} \rightarrow H_{2}+\Sigma_{2} \text {. }
$$

Then, the energies and wave functions of low-lying states are determined by diagonalizing the effective Hamiltonian:

$$
H^{\mathrm{eff}}=H_{1}+H_{2},
$$

where $H_{1}$ and $H_{2}$ are modified according to Eqs. (2) and (3). The matrix elements and other properties, such as polarizabilities, can be determined using the resulting wave functions 27.

In the CI + all-order approach, the corrections to the effective Hamiltonian $\Sigma_{1}$ and $\Sigma_{2}$ are calculated using a modified version of the linearized coupled-cluster all-order method which allows to include dominant core and core-valence correlation corrections to the effective Hamiltonian to all orders and improve accuracy in comparison with the CI+MBPT method. The detailed description of the CI+all-order method and all formulas are given in 26].

When the CI space includes only two or three electrons, it can be made essentially complete. For four-electron systems, we have developed an efficient algorithm to construct a sufficiently complete set of configurations. The $\mathrm{CI}+$ all-order method yielded accurate wave functions for calculations of such atomic properties as lifetimes, polarizabilities, hyperfine structure constants, etc, for a number of divalent and threvalent systems [26, 29 35]. The spectra of the superheavy elements No, Lr and Rf with two, three, and four valence electrons were recently presented by Dzuba et al. [36].

We included the Breit interaction on the same footing as the Coulomb interaction at the stage of constructing the basis set, and incorporated the Gaunt part of the Breit interaction in the CI. The QED radiative corrections to the energy levels are included using the method described in [37]. We find the QED contribution to be significant only for the configurations that contain valence $5 s$ state, and omit it for Sn-like ions where none of 
the low-lying configurations contain $5 s$ valence electron. The partial waves with $l_{\max }=6$ are included in all summations in many-body perturbation theory or coupledcluster terms. Extrapolation of the $l>6$ contribution is carried out following the method described in Ref. [19].

The lifetime of a state $a$ is calculated as

$$
\tau_{a}=\frac{1}{\sum_{b} A_{a b}},
$$

where the multipole transition rates $A_{a b}$ are related to the line strengths $S_{a b}$. Explicit expressions are given in Ref. [19]. In the sum (5) we account for the electric $(E k)$ and magnetic $(M k)$ transitions of the ranks $k=1-3$.

The sensitivity of the atomic transition frequency $\omega$ to the variation of the fine-structure constant $\alpha$ can be quantified using a coefficient $q$ defined as $\omega(x)=\omega_{0}+q x$, where $x \equiv\left(\frac{\alpha}{\alpha_{0}}\right)^{2}-1$ and the frequency $\omega_{0}$ corresponds to the value of the fine-structure constant $\alpha_{0}$ at some initial point in time. It is convenient to also define dimensionless enhancement factor $K=2 q / \omega$. We follow the same procedure to calculate $q$ as in Ref. [19]. Briefly, we carry out three calculations with different values of $\alpha$ for every ion considered in this work. In the first calculation, current CODATA value of $\alpha$ [38] is used. In the other two calculations, the value of $\alpha^{2}$ is varied by $\pm 1 \%$. The value of $q$ is then determined as a numerical derivative.

\section{CD-LIKE IONS}

The $5 s-4 f$ level crossing in Cd-like ions happens for $\mathrm{Nd}^{12+}-\mathrm{Sm}^{14+}$ ions. The order of levels in previous ions of the Cd-like isoelectronic sequence, such as $\mathrm{Ba}^{8+}$, is $5 s^{2}, 5 s 5 p$, and $5 s 4 f$. It changes to $5 s^{2}, 5 s 4 f$, and $5 s 5 p$ for $\mathrm{Nd}^{12+}$. The $4 f^{2}$ becomes the ground state for $\mathrm{Sm}^{14+}$, with other low-lying levels belonging to either $4 f^{2}$ or $5 s 4 f$ configurations. In order to evaluate the uncertainties of our values, we carried out several calculations which allowed us to separate the effect of higher orders, Breit interaction, contributions of higher partial waves, and QED. The contribution of the higher orders is evaluated as the difference of the CI+all-order and CI+MBPT results. The Breit and QED contributions are calculated as the difference of the results with and without the inclusion of these effects. The contribution of the higher $(l>6)$ partial waves (labeled "Extrap") is estimated to be equal to the contribution of the $l=6$ partial wave following our empiric rule obtained for Ag-like ions (see [19] for a detailed discussion of the extrapolation). The contribution of the $l=6$ partial wave is obtained as the difference of two calculations where all intermediate sums in the all-order and MBPT terms are restricted to $l_{\max }=6$ and $l_{\max }=5$. The resulting four contributions are listed separately in Table 【. The final theoretical results are listed in "Final" column.

We develop several methods to estimate the accuracy of our calculations. First, we assume that the uncertainty of each of the four corrections (HO, Extrap, Breit, and QED) does not exceed $25 \%$, and add $25 \%$ of each correction in quadrature to estimate the total uncertainty. In Ag-like and In-like ions, such estimates are significantly larger (by a factor 2-4) than our actual difference with the experiment for all three ions listed in Table I of [18]. For $\mathrm{Ba}^{+8} 5 s 4 f$ states, which are of most interest for the present work, such estimate gives about $400 \mathrm{~cm}^{-1}$, while our differences with experiment are $70-270 \mathrm{~cm}^{-1}$. Therefore, we can expect that such procedure will give reasonable estimates of uncertainties for the $5 s 4 f$ states of $\mathrm{Nd}^{12+}$.

In the second approach of evaluating the uncertainties, we use the reference ion, $\mathrm{Ba}^{8+}$, to estimate the uncertainties in the calculations for the other ion. We estimate the uncertainty as the sum of the following: (1) difference of the theoretical and experimental energies for the reference ion and (2) difference in the sum of all four corrections between the reference and the current ion. For the $5 s 5 p$ states of $\mathrm{Nd}^{12+}$, we use this second approach to estimate the uncertainties and also find that these estimates are significantly smaller than our difference with the experiment.

The agreement of the $5 s 4 f$ energies with the experiment 20] for $\mathrm{Ba}^{8+}$ is excellent and is of the same relative magnitude $(0.1 \%)$ as in the case of $4 f$ states of Ag-like $\mathrm{Ba}^{9+}$ ion. The $5 s 5 p$ energies agree with experiment to about $0.6 \%$. However, the differences with the experiment for $\mathrm{Nd}^{12+}$ 24] energies are anomalously large, 1.6$3 \%$ for all states listed in Table \except $5 s 5 p{ }^{1} P_{1}$ which is in excellent agreement with experiment. These differences are much larger than our estimated upper bound on the uncertainty of our results listed in column "Unc".

To explore the discrepancy of our energies with experiment for $\mathrm{Nd}^{12+}$, we calculated the energies of the other three ions of Cd-like isoelectronic sequence, $\mathrm{La}^{9+}, \mathrm{Ce}^{10+}$, and $\mathrm{Pr}^{11+}$, and compared the results with the experimental values from [21 23]. We present the comparison of theoretical energies with experiment for all 5 consecutive ions of Cd-like isoelectronic sequence, $\mathrm{Ba}^{8+}$ [20], $\mathrm{La}^{9+}$ [21], $\mathrm{Ce}^{10+}$ 22], $\mathrm{Pr}^{11+}$ 23], and $\mathrm{Nd}^{12+}$ 24] in TableII All energies are given relative to the $5 s^{2}{ }^{1} S_{0}$ ground state in $\mathrm{cm}^{-1}$. Actual (in $\mathrm{cm}^{-1}$ ) and relative (in \%) differences with experiment are given for all states. The states are listed in the same order for all 5 ions for the convenience of presentation. The actual order of states starts to change for $\mathrm{Ce}^{10+}$. The fine structure splittings of the $5 s 5 p$ and $4 f 5 s$ triplets are listed in the last four rows. Table II clearly illustrates the abrupt shift in the agreement with experiment between the first three and last two ions. It appears that all levels with the exception of the $5 s 5 p^{1} P_{1}$ suddenly shift by about $2000 \mathrm{~cm}^{-1}$ for $\operatorname{Pr}^{11+}$. We note that the $5 s^{2}{ }^{1} S_{0}-5 s 5 p{ }^{1} P_{1}$ is the only strong easily identifiable line from all of the states considered here. The identification of other numerous ultraviolet (UV) lines is a very difficult task carried out in [21 23] using the Cowan code. It may be possible that change in the order of levels for $\operatorname{Pr}^{11+}$ resulted in 
TABLE I: Energies of Cd-like $\mathrm{Ba}^{8+}, \mathrm{Nd}^{12+}$, and $\mathrm{Sm}^{14+}$ ions relative to their ground states evaluated using the CI+all-order method (in $\mathrm{cm}^{-1}$ ). Contributions from higher-order Coulomb correlation (difference of the CI+all-order and CI+MBPT calculation), estimated contributions of higher partial waves $(l>6)$, the Breit and QED corrections are given separately in columns HO, Extrap, Breit, and QED. Experimental results are from [20] for $\mathrm{Ba}^{8+}$ and [24] for $\mathrm{Nd}^{12+}$. Difference with experiment is given in $\mathrm{cm}^{-1}$ and \% in columns "Diff." Estimated absolute uncertainties of theoretical calculations are given in columns "Unc". Theoretical and experimental wavelengths for transitions to the ground states are given in last two columns in $\mathrm{nm}$.

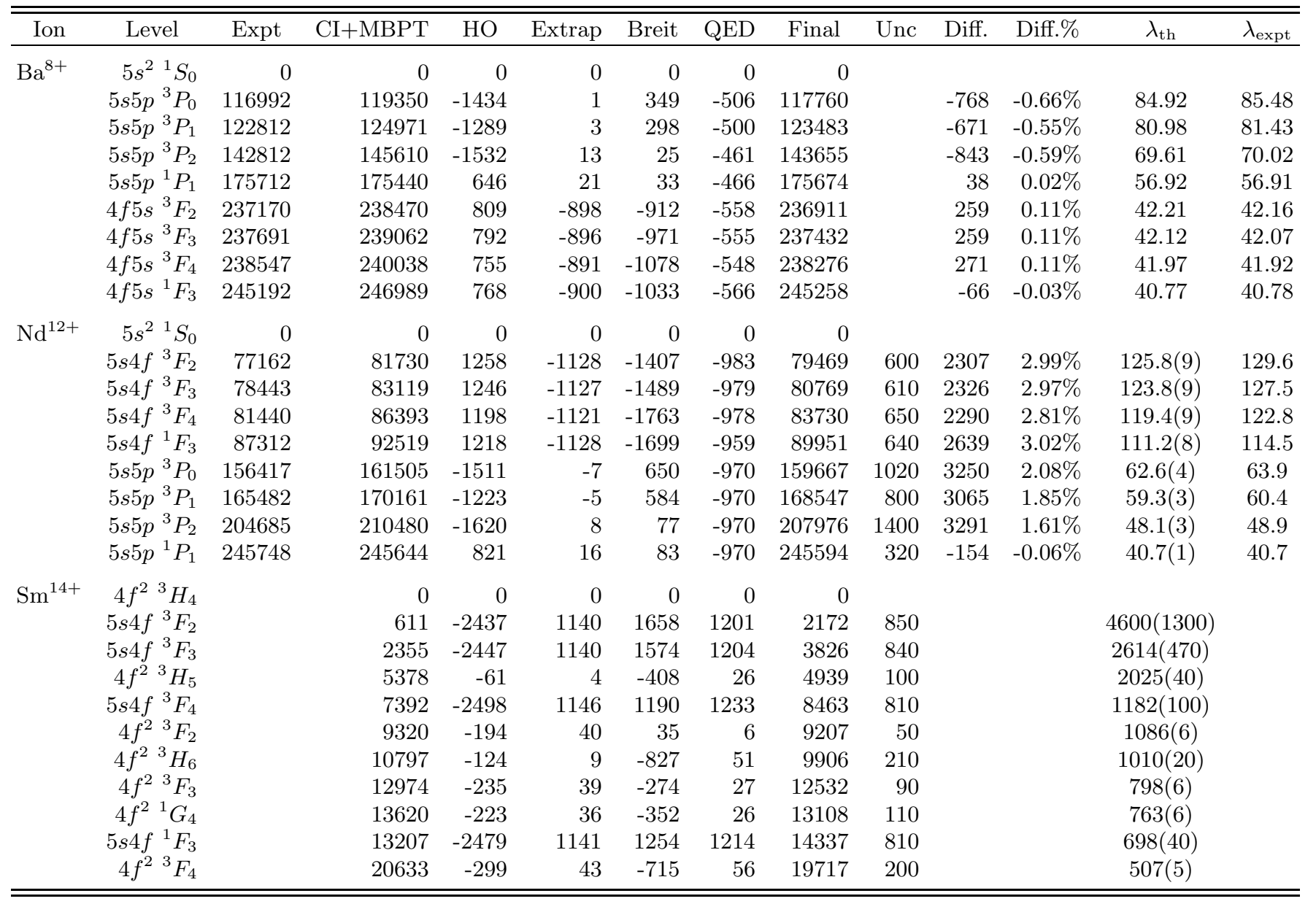

TABLE II: Comparison of theoretical energies with experiment for Cd-like $\mathrm{Ba}^{8+}$ [20], $\mathrm{La}^{9+}[21], \mathrm{Ce}^{10+}$ [22], $\mathrm{Pr}^{11+}$ [23], and $\mathrm{Nd}^{12+}$ [24] ions relative to the $5 s^{2}{ }^{1} S_{0}$ ground state $\left(\right.$ in $\left.\mathrm{cm}^{-1}\right)$. Actual (in $\mathrm{cm}^{-1}$ ) and relative (in \%) differences with experiment are given for all states. The states are listed in the same order for all ions. Fine structure intervals for $5 s 5 p$ and $4 f 5 s$ triplets are listed in the last four rows.

\begin{tabular}{|c|c|c|c|c|c|c|c|c|c|c|c|c|c|c|c|}
\hline \multirow[t]{2}{*}{ Level } & \multicolumn{3}{|c|}{$\mathrm{Ba}^{8+}$} & \multicolumn{3}{|c|}{$\mathrm{La}^{9+}$} & \multicolumn{3}{|c|}{$\overline{C e^{10+}}$} & \multicolumn{3}{|c|}{$\overline{P r^{11+}}$} & \multicolumn{3}{|c|}{$\mathrm{Nd}^{12+}$} \\
\hline & Theory & Diff. & $\%$ & Theory & Diff. & $\%$ & Theory & Diff. & $\%$ & Theory & Diff. & $\%$ & Theory & Diff. & $\%$ \\
\hline $5 s 5 p^{3} P_{0}$ & 117760 & -768 & -0.7 & 128226 & -811 & -0.6 & 138718 & -867 & -0.6 & 149252 & -2934 & -2.0 & 159667 & -3250 & -2.1 \\
\hline $5 s 5 p{ }^{3} P_{1}$ & 23483 & -671 & -0.6 & 134719 & -697 & -0.5 & 145996 & -729 & -0.5 & 157329 & -2774 & -1.8 & 168547 & -3065 & -1.9 \\
\hline $5 s 5 p{ }^{3} P_{2}$ & 143655 & -843 & -0.6 & 158956 & -885 & -0.6 & 173838 & -891 & -0.5 & 191188 & -3020 & -1.6 & 207976 & -3291 & -1.6 \\
\hline $5 s 5 p{ }^{1} P_{1}$ & 175674 & 38 & 0.0 & 192451 & 33 & 0.0 & 209702 & 1 & 0.0 & 227471 & -24 & -0.0 & 245594 & 154 & 0.1 \\
\hline $4 f 5 s{ }^{3} F_{2}$ & 236911 & 259 & 0.1 & 207147 & 165 & 0.1 & 170661 & 41 & 0.0 & 127955 & -1969 & -1.6 & 79469 & -2307 & -3.0 \\
\hline $4 f 5 s{ }^{3} F_{3}$ & 237432 & 259 & 0.1 & 207865 & 159 & 0.1 & 171521 & 91 & 0.1 & 129067 & -1984 & -1.6 & 80769 & -2326 & -3.0 \\
\hline $4 f 5 s{ }^{3} F_{4}$ & 238276 & 271 & 0.1 & 209118 & 171 & 0.1 & 173364 & 104 & 0.1 & 131378 & -1964 & -1.5 & 83730 & -2290 & -2.8 \\
\hline $4 f 5 s{ }^{1} F_{3}$ & 245258 & -66 & -0.0 & 215933 & -144 & -0.1 & 179860 & -232 & -0.1 & 137767 & -2297 & -1.7 & 89951 & -2639 & -3.0 \\
\hline${ }^{3} P_{1}-{ }^{3} P_{0}$ & 5723 & 97 & 1.7 & 6493 & 114 & 1.7 & 7278 & 138 & 1.9 & 8077 & 160 & 1.9 & 8880 & 185 & 2.0 \\
\hline${ }^{3} P_{2}-{ }^{3} P_{1}$ & 20172 & -172 & -0.9 & 24237 & -188 & -0.8 & 28571 & -162 & -0.6 & 33859 & -246 & -0.7 & 39429 & -226 & -0.6 \\
\hline${ }^{3} F_{3}-{ }^{3} F_{2}$ & 521 & 0 & 0.0 & 718 & -6 & -0.8 & 860 & 50 & 5.5 & 1112 & -15 & -1.4 & 1300 & -19 & -1.5 \\
\hline${ }^{3} F_{4}-{ }^{3} F_{3}$ & 844 & 12 & 1.4 & 1253 & 12 & 1.0 & 1752 & 13 & 0.7 & 2311 & 20 & 0.9 & 2961 & 36 & 1.2 \\
\hline
\end{tabular}


TABLE III: Transition energies $\omega$ and sensitivity coefficients $q$ for Cd-like ions relative to the ground state evaluated using the CI+all-order method in $\mathrm{cm}^{-1} ; K=2 q / \omega$ is the enhancement factor.

\begin{tabular}{crrrr}
\hline \hline Ion & \multicolumn{1}{c}{ Level } & \multicolumn{1}{c}{$\omega$} & \multicolumn{1}{c}{$q$} \\
\hline $\mathrm{Nd}^{12+}$ & $5 s^{2}{ }^{1} S_{0}$ & 0 & 0 & \\
& $5 s 4 f{ }^{3} F_{2}$ & 79469 & 101461 & 2.6 \\
& $5 s 4 f{ }^{3} F_{3}$ & 80769 & 102325 & 2.5 \\
& $5 s 4 f{ }^{3} F_{4}$ & 83730 & 105340 & 2.5 \\
& $5 s 4 f{ }^{1} F_{3}$ & 89951 & 105827 & 2.4 \\
& $5 s 5 p{ }^{3} P_{0}$ & 159667 & 14175 & 0.2 \\
& $5 s 5{ }^{3} P_{1}$ & 168547 & 19465 & 0.2 \\
$\mathrm{Sm}^{14+}$ & $4 f^{2}{ }^{3} H_{4}$ & 0 & 0 & \\
& $5 s 4 f{ }^{3} F_{2}$ & 2172 & -127720 & -118 \\
& $5 s 4 f{ }^{3} F_{3}$ & 3826 & -126746 & -66 \\
& $4 f^{2}{ }^{3} H_{5}$ & 4939 & 4917 & 2.0 \\
$5 s 4 f{ }^{3} F_{4}$ & 8463 & -121952 & -29 \\
$4 f^{2}{ }^{3} F_{2}$ & 9207 & 1324 & 0.3 \\
& $4 f^{2}{ }^{3} H_{6}$ & 9906 & 9295 & 1.9 \\
$4 f^{2}{ }^{3} F_{3}$ & 12532 & 4954 & 0.8 \\
& $4 f^{2}{ }^{1} G_{4}$ & 13108 & 4508 & 0.7 \\
& $5 s 4 f{ }^{1} F_{3}$ & 14337 & -121525 & -17 \\
$4 f^{2}{ }^{3} F_{4}$ & 19717 & 10045 & 1.0 \\
\hline \hline
\end{tabular}

some identification problem. Since our calculations are carried out in the same way for all ions, we find abrupt $2000 \mathrm{~cm}^{-1}$ shift in accuracy to be unlikely. Further measurements are needed to resolve this problem. We use the first (25\%) approach to estimate the accuracy of our calculations for $\mathrm{Sm}^{14+}$ energies since $4 f^{2}$ configuration is not present among measured low-lying levels of $\mathrm{Ba}^{8+}$, and this reference ion cannot be used for $\mathrm{Sm}^{14+}$.

The CI+all-order sensitivity coefficients $q$ for Cd-like ions obtained as described in Section [I] are given in Table III All energy and $q$ values are given relative to the ground state in $\mathrm{cm}^{-1}$. The CI+all-order energies and $q$ coefficients are used to calculate enhancement factors $K=2 q / \omega$ given in the last row of the table. The enhancement factors are very large for all transitions from the $5 s 4 f$ levels to the ground state for $\mathrm{Sm}^{14+}$ due to large $q$ and small transition energies. The calculation of $q$ for Ag-like ions [19] demonstrated that the effect of correlation is small for the cases where $q$ are large, i.e. all cases of interest. Therefore, the uncertainties in large values of $K$ will be dominated by the uncertainties in the transition energies, in particular where they exceed 2-3\%. Then, the relative uncertainty in $K$ for $4 f^{2}{ }^{3} H_{4}-5 s 4 f$ transitions can be estimated as the relative uncertainty in the corresponding transition energy. We note that $q$ values are positive for $\mathrm{Nd}^{12+}$ and negative for $\mathrm{Sm}^{14+}$. This creates additional enhancements for $\alpha$ variation search if the relative transition frequencies in $\mathrm{Nd}^{12+} / \mathrm{Sm}^{14+}$ are monitored.

The CI+all-order multipole reduced matrix elements $Z$, transition rates $A_{r}$, and lifetimes $\tau$ in Cd-like $\mathrm{Nd}^{12+}$ and $\mathrm{Sm}^{14+}$ ions are given in Table IV] We use theoret- ical energies in transition rate and lifetime calculations. The numbers in brackets represent powers of 10 . The strongest transition from the first excited levels of both ions is $M 2$, leading to the extremely long lifetimes. The case of $\mathrm{Nd}^{12+}$ is very similar to $\mathrm{Ag}$-like $\mathrm{Nd}^{13+}$ discussed in Ref. [19] but the wavelengths are further in UV. Next excited states in both ions live also very long, with $20 \mathrm{~s}$ and $8.5 \mathrm{~s}$ lifetimes for $\mathrm{Nd}^{12+}$ and $\mathrm{Sm}^{14+}$, respectively.

\section{SN-LIKE IONS}

The Sn-like ions, considered in this work, may be treated either as divalent systems with $1 s^{2} 2 s^{2} 2 p^{6} 3 s^{2} 3 p^{6} 3 d^{10} 4 s^{2} 4 p^{6} 4 d^{10} 5 s^{2}$ core or systems with four valence electrons (then, the $5 s$ electrons are in the valence field). We carry out both calculations to ensure that all dominant configurations are taken into account. We refer to the results of these calculations as 2-val and 4-val, respectively. We carried out the same calculations for the $\mathrm{Ba}^{6+}$ ion, which is the last ion in Sn isoelectronic sequence where experimental energies are available. Unfortunately, the experimental data for this ion are limited to the fine-structure of the $5 p^{2}$ configuration. The results of 2-val and 4-val calculations for Sn-like ions are summarized in Table $\mathrm{V}$ where we list the energies of Sn-like $\mathrm{Ba}^{6+}, \mathrm{Pr}^{9+}$, and $\mathrm{Nd}^{10+}$ ions relative to the ground state. Contributions from higher-order Coulomb correlation (difference of the CI+all-order and $\mathrm{CI}+\mathrm{MBPT}$ calculations), estimated contributions of higher partial waves $(l>6)$, and Breit interaction are given separately in columns labeled "HO", "Extrap", and "Breit". QED contribution is considered to be negligible for these states.

We find a technical complication in applying CI+allorder method to the Sn-like ions. Both the CI+MBPT and $\mathrm{CI}+$ all-order methods are based on the BrilloiunWigner variant of the MBPT, rather than the RayleighSchrödinger to avoid nonsymmetric effective Hamiltonian and the problem of intruder states. In the BrilloiunWigner variant of MBPT, the effective Hamiltonian is symmetric and accidentally small denominators do not arise, but the many-body corrections to the Hamiltonian $\Sigma_{1}$ and $\Sigma_{2}$ become energy dependent. Solving the equation for $H^{\text {eff }}$ we are able to find these energies. But since we use the single-particle perturbation theory, more simple and practical approach is to set this energy, $\tilde{\varepsilon}$, to be the Dirac-Fock energy of the lowest orbital for the particular partial wave (see Refs. 26, 28] for more details).

For all Cd-like calculations carried out in this work, this approach works perfectly fine. However, we find that the use of the lowest $5 d$ energies, as $\tilde{\epsilon}$, is not appropriate for the 2-val calculations for the Sn-like ions. This is because the $5 s$ state, which is treated as a core state in 2-val calculations, has small excitation energy, very close to the excitation energy of the $5 d$ state. This leads to extremely small energy denominator in the expression for $\hat{\Sigma}$, e. g., $\varepsilon_{5 s}+\varepsilon_{5 d}-\varepsilon_{5 p}-\varepsilon_{5 p} \simeq 0$. This means that the sum of two 
TABLE IV: The CI+all-order multipole reduced matrix elements $Z$ (in a.u.), transition rates $A_{r}$ (in s ${ }^{-1}$ ), and lifetimes $\tau$ (in sec) in Cd-like $\mathrm{Nd}^{12+}$ and $\mathrm{Sm}^{14+}$ ions. Transition energies (in $\mathrm{cm}^{-1}$ ) and corresponding wavelengths (in nm) are obtained from the theoretical energies given in Table [ The numbers in brackets represent powers of 10.

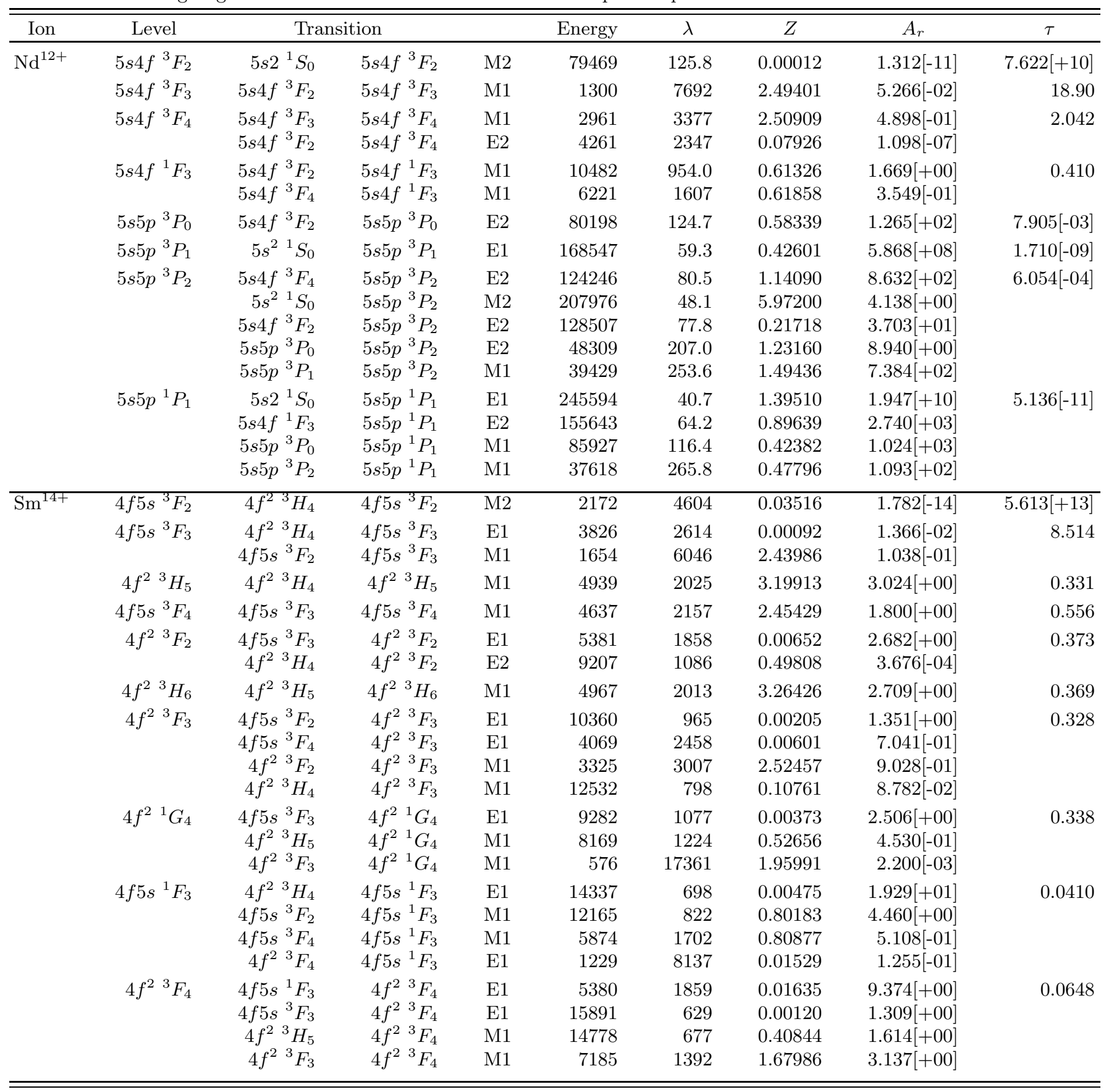

single-electron energies $\varepsilon_{5 s}+\varepsilon_{5 d}$ is a poor approximation for the two-electron energies of low states of Sn-like ions which must enter the expression for $\hat{\Sigma}$. Therefore, we use the $5 p_{1 / 2}$ energies for $\tilde{\varepsilon}$ in the expressions for $\hat{\Sigma}$ operator for $n s$ and $n d$ states. The $4 f_{j}$ energies are used for the $n f$ states.

We have tested the sufficient completeness of the fourelectron configuration space by carrying out three calculations with increasing number of configurations. The first run contained only double excitations into the valence space from a few main configurations. Two larger runs were selectively constructed by allowing extra excitations into the valence space from several hundreds most important configurations. Thus, triple and quadrupole excitations from initial configurations were effectively included. The differences between the results of first two runs were less then $350 \mathrm{~cm}^{-1}$. The differences between second and final largest runs with 23000 and 32000 con- 
TABLE V: Comparison of CI+all-order energies of Sn-like $\mathrm{Ba}^{6+}, \mathrm{Pr}^{9+}$, and $\mathrm{Nd}^{10+}$ ions relative to the ground state calculated as two-valence-electron (2-valence) and four-valence-electron (4-valence) systems (in $\mathrm{cm}^{-1}$ ). In the two-valence electron calculation, the $5 \mathrm{~s}$ shell is taken to be a core shell. Contributions from higher-order Coulomb correlation (difference of the $\mathrm{CI}+$ all-order and CI+MBPT calculations), estimated contributions of higher partial waves $(l>6)$, and Breit corrections are given separately in columns labeled "HO", "Extrap", and "Breit". Differences between "4-valence" and "2-valence" final values are given in last column.

\begin{tabular}{|c|c|c|c|c|c|c|c|c|c|c|c|c|c|}
\hline \multirow[b]{2}{*}{ Ion } & \multirow[b]{2}{*}{ Term } & \multirow[b]{2}{*}{$J$} & \multicolumn{5}{|c|}{ 2-valence calculation } & \multicolumn{4}{|c|}{ 4-valence calculation } & \multirow[b]{2}{*}{ Final } & \multirow[t]{2}{*}{ Diff. } \\
\hline & & & $\mathrm{CI}+\mathrm{MBPT}$ & $\mathrm{HO}$ & Extrap & Breit & Final & $\mathrm{CI}+\mathrm{MBPT}$ & $\mathrm{HO}$ & Extrap & Breit & & \\
\hline \multirow[t]{5}{*}{$\mathrm{Ba}^{6+}$} & $5 p^{2}$ & ${ }^{3} P_{0}$ & 0 & 0 & 0 & 0 & 0 & 0 & 0 & 0 & 0 & 0 & 0 \\
\hline & $5 p^{2}$ & ${ }^{3} P_{1}$ & 15554 & 153 & 13 & -242 & 15477 & 15704 & -103 & 10 & -238 & 15372 & -105 \\
\hline & $5 p^{2}$ & ${ }^{3} P_{2}$ & 21228 & 187 & 13 & -267 & 21161 & 21788 & -108 & 9 & -268 & 21422 & 261 \\
\hline & $5 p^{2}$ & ${ }^{1} D_{2}$ & 42400 & -26 & 24 & -510 & 41888 & 43143 & -233 & 17 & -507 & 42420 & 532 \\
\hline & $5 p^{2}$ & ${ }^{1} S_{0}$ & 62976 & -1466 & 25 & -505 & 61030 & 62342 & -260 & 21 & -501 & 61602 & 572 \\
\hline \multirow[t]{8}{*}{$\operatorname{Pr}^{9+}$} & $5 p^{2}$ & ${ }^{3} P_{0}$ & 0 & 0 & 0 & 0 & 0 & 0 & 0 & 0 & 0 & 0 & U \\
\hline & $5 p 4 f$ & ${ }^{3} G_{3}$ & 20050 & 2994 & -1078 & -1750 & 20216 & 21865 & 2810 & -1032 & -1748 & 21895 & 1679 \\
\hline & $5 p 4 f$ & ${ }^{3} F_{2}$ & 22664 & 2489 & -862 & -1519 & 22772 & 24172 & 2291 & -829 & -1435 & 24199 & 1427 \\
\hline & $5 p 4 f$ & ${ }^{3} F_{3}$ & 25607 & 2844 & -1072 & -2017 & 25362 & 27233 & 2804 & -1026 & -2009 & 27002 & 1640 \\
\hline & $5 p 4 f$ & ${ }^{3} F_{4}$ & 27727 & 2943 & -1080 & -2054 & 27536 & 29622 & 2801 & -1033 & -2048 & 29343 & 1806 \\
\hline & $5 p^{2}$ & ${ }^{3} P_{1}$ & 28712 & 193 & 16 & -409 & 28512 & 28962 & -135 & 14 & -405 & 28436 & -76 \\
\hline & $5 p^{2}$ & ${ }^{3} P_{2}$ & 35831 & 856 & -252 & -782 & 35653 & 36697 & 615 & -243 & -852 & 36217 & 564 \\
\hline & $5 p 4 f$ & ${ }^{1} F_{3}$ & 54104 & 2728 & -1066 & -2179 & 53588 & 55735 & 2680 & -1023 & -2172 & 55220 & 1632 \\
\hline \multirow[t]{8}{*}{$\mathrm{Nd}^{10+}$} & $4 f^{2}$ & ${ }^{3} H_{4}$ & 0 & 0 & 0 & 0 & 0 & 0 & 0 & 0 & 0 & 0 & 0 \\
\hline & $5 p 4 f$ & ${ }^{5} G_{3}$ & 2605 & -4037 & 1076 & 1920 & 1564 & 1953 & -4305 & 1025 & 1887 & 560 & -1004 \\
\hline & $4 f^{2}$ & ${ }^{3} H_{5}$ & 3432 & -80 & 1 & -294 & 3059 & 3405 & -58 & 2 & -291 & 3058 & -1 \\
\hline & $5 p 4 f$ & ${ }^{1} D_{2}$ & 5975 & -3629 & 1009 & 1704 & 5060 & 5171 & -3823 & 923 & 1768 & 4040 & -1020 \\
\hline & $4 f^{2}$ & ${ }^{3} H_{6}$ & 6982 & -167 & 6 & -599 & 6222 & 6930 & -128 & 8 & -590 & 6219 & -3 \\
\hline & $5 p 4 f$ & ${ }^{3} F_{3}$ & 8448 & -3861 & 1037 & 1471 & 7095 & 7853 & -4222 & 1063 & 1687 & 6382 & -713 \\
\hline & $4 f^{2}+5 p 4 f$ & ${ }^{3} F_{2}$ & 8263 & -694 & 113 & 231 & 7914 & 8323 & -594 & 144 & 134 & 8007 & 93 \\
\hline & $4 f^{2}+5 p 4 f$ & ${ }^{5} G_{4}$ & 9391 & -3048 & 908 & 1102 & 8353 & 8845 & -3330 & 801 & 1309 & 7624 & -729 \\
\hline
\end{tabular}

TABLE VI: Comparison of the CI+all-order energies of Snlike $\mathrm{Ba}^{6+}$ relative to the ground state calculated as twovalence-electron (2-val) and four-valence-electron (4-val) system $\left(\right.$ in $\left.\mathrm{cm}^{-1}\right)$. Experimental results 20] are listed in column labeled "Expt.". The columns $\Delta_{2 \mathrm{val}}$ and $\Delta_{4 \mathrm{val}}$ give differences between 2-val and 4-val calculations and experiment. In the two-valence-electron calculation, the $5 s$ shell is taken to be a core shell. The column labeled "Ave." gives average of the 4 -val and 2-val calculations. In last column the difference of averaged results with experiment is presented.

\begin{tabular}{|c|c|c|c|c|c|c|c|}
\hline Level & 2-val & 4-val & Expt. & $\Delta_{2 \mathrm{val}}$ & $\Delta_{4 \mathrm{val}}$ & Ave. & $\Delta_{\text {Ave }}$ \\
\hline $5 p^{2}{ }^{3} P_{0}$ & 0 & 0 & 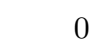 & 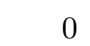 & 0 & 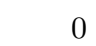 & U \\
\hline $5 p^{2}{ }^{3} P_{1}$ & 15477 & 15372 & 15507 & 30 & 135 & 15425 & 82 \\
\hline $5 p^{2}{ }^{3} P_{2}$ & 21161 & 21422 & 21499 & 338 & 77 & 21291 & 208 \\
\hline $5 p^{2}{ }^{1} D_{2}$ & 41888 & 42420 & 42514 & 626 & 94 & 42154 & 360 \\
\hline $5 p^{2}{ }^{1} S_{0}$ & 61030 & 61602 & 61083 & 53 & -519 & 61316 & -233 \\
\hline
\end{tabular}

figurations, respectively, were less than $10 \mathrm{~cm}^{-1}$ indicating sufficient saturation of the configuration space.

We provide a detailed comparison of 2-val and 4-val results with the experiment for $\mathrm{Ba}^{6+}$ [20] in Table VI] While we find a good agreement of both results with experiment, it is unclear whether 2-val or 4-val calculations provide better accuracy. The agreement with experiment is different for the four states. Most likely this is caused by the admixture of configurations that cannot be described as divalent $5 s^{2} n l n^{\prime} l^{\prime}$ states. It appears that the average of both calculations produces the results that are the most consistent with experiment for all states. Unfortunately, we have no comparison with experiment for the $5 p 4 f$ and $4 f^{2}$ configurations which are of interest for the present work.

The ions of interest in the Sn-like isoelectronic sequence are $\operatorname{Pr}^{9+}$ and $\mathrm{Nd}^{10+}$ where the $5 p^{2}$ and $5 p 4 f$ or $5 p 4 f$ and $4 f^{2}$ levels become very close due to the $5 p-4 f$ level crossing. The case of $\operatorname{Pr}^{9+}$ is particularly interesting, since the several lowest metastable levels have transitions to the ground state in the optical range. The ground and first excited states of $\mathrm{Nd}^{10+}$ are extremely close and the resulting uncertainty is on the order of the transition energy. While our calculations place $4 f^{2}$ to be the ground state, the higher-order corrections are particularly large in this case, almost 3 times that of the transition energy, which might lead to the placement of the $5 p 4 f J=3$ as the ground state.

Determination of the uncertainties is difficult for these ions due to complete lack of data for comparison. We also observe strong cancellations between various large corrections. Therefore, adding $25 \%$ of all corrections in quadrature may significantly overestimate the uncertain- 
TABLE VII: Comparison of the CI+all-order energies of Sn-like $\mathrm{Pr}^{9+}$ and $\mathrm{Nd}^{10+}$ relative to the ground state calculated as two-valence-electron (2-val) and four-valence-electron (4-val) system (in $\mathrm{cm}^{-1}$ ). The final numbers, which are the average of two calculations are listed in the column labeled "Final". Estimated absolute uncertainties of the respective values are given in columns "Unc" in $\mathrm{cm}^{-1}$. Wavelengths for transitions to the ground state and their uncertainties (in parenthesis) are given in last column in $\mathrm{nm}$.

\begin{tabular}{|c|c|c|c|c|c|c|c|c|c|}
\hline Ion & Term & $J$ & 2-val & Unc. & 4-val & Unc. & Final & Unc. & $\lambda$ \\
\hline \multirow[t]{5}{*}{$\operatorname{Pr}^{9+}$} & $5 p^{2}$ & ${ }^{3} P_{0}$ & 0 & & 0 & 0 & 0 & 0 & \\
\hline & $5 p 4 f$ & ${ }^{3} G_{3}$ & 20216 & 540 & 21895 & 450 & 21055 & 840 & $475(18)$ \\
\hline & $5 p 4 f$ & ${ }^{3} F_{3}$ & 25362 & 580 & 27002 & 570 & 26182 & 820 & $382(12)$ \\
\hline & $5 p 4 f$ & ${ }^{3} F_{4}$ & 27536 & 560 & 29343 & 590 & 28440 & 900 & $352(11)$ \\
\hline & $5 p^{2}$ & ${ }^{3} P_{1}$ & 28512 & 160 & 28436 & 320 & 28474 & 320 & $351.2(5)$ \\
\hline \multirow[t]{5}{*}{$\mathrm{Nd}^{10+}$} & $4 f^{2}$ & ${ }^{3} \mathrm{H}_{4}$ & 0 & 0 & 0 & 0 & 0 & 0 & \\
\hline & $5 p 4 f$ & ${ }^{5} G_{3}$ & 1564 & 1100 & 560 & 1300 & 1062 & 1300 & \\
\hline & $4 f^{2}$ & ${ }^{3} H_{5}$ & 3059 & 220 & 3058 & 210 & 3059 & 220 & $3270(220)$ \\
\hline & $5 p 4 f$ & ${ }^{1} D_{2}$ & 5060 & 980 & 4040 & 1100 & 4550 & 1100 & $2200(430)$ \\
\hline & $4 f^{2}$ & ${ }^{3} H_{6}$ & 6222 & 460 & 6219 & 430 & 6221 & 460 & $1610(110)$ \\
\hline
\end{tabular}

TABLE VIII: Transition energies, $\omega$, and sensitivity coefficients $q$ for Sn-like ions relative to the ground state evaluated in the CI+all-order approximation in $\mathrm{cm}^{-1} ; K=2 q / \omega$ is the enhancement factor.

\begin{tabular}{crrrrr}
\hline \hline Ion & \multicolumn{1}{c}{ Term } & $J$ & $\omega$ & $q$ & $K$ \\
\hline $\operatorname{Pr}^{9+}$ & $5 p^{2}$ & ${ }^{3} P_{0}$ & 0 & 0 & \\
& $5 p 4 f$ & ${ }^{3} G_{3}$ & 20216 & 42721 & 4.2 \\
& $5 p 4 f$ & ${ }^{3} F_{2}$ & 22772 & 42865 & 3.8 \\
& $5 p 4 f$ & ${ }^{3} F_{3}$ & 25362 & 47076 & 3.7 \\
$5 p 4 f$ & ${ }^{3} F_{4}$ & 27536 & 37197 & 2.7 \\
& $5 p^{2}$ & ${ }^{3} P_{1}$ & 28512 & 47483 & 3.3 \\
$\mathrm{Nd}^{10+}$ & $4 f^{2}$ & ${ }^{3} H_{4}$ & 0 & & \\
& $5 p 4 f$ & ${ }^{5} G_{3}$ & 1564 & -81052 & -104 \\
& $4 f^{2}$ & ${ }^{3} H_{5}$ & 3059 & 3113 & 2.0 \\
& $5 p 4 f$ & ${ }^{1} D_{2}$ & 5060 & -60350 & -24 \\
$4 f^{2}$ & ${ }^{3} H_{6}$ & 6222 & 5930 & 1.9 \\
& $5 p 4 f$ & ${ }^{3} F_{3}$ & 7095 & -63285 & -18 \\
& $4 f^{2}+5 p 4 f$ & ${ }^{3} F_{2}$ & 7914 & -17809 & -4.5 \\
& $4 f^{2}+5 p 4 f$ & ${ }^{5} G_{4}$ & 8353 & -39672 & -9.5 \\
\hline \hline
\end{tabular}

ties. We take the average of the $25 \%$ estimate and the total sum of all corrections as an uncertainty for all levels and list these values. The uncertainties are independently evaluated for 2-val and 4-val calculations following this prescription. Comparison of the CI+all-order energies of Sn-like $\mathrm{Pr}^{9+}$ and $\mathrm{Nd}^{10+}$ relative to the ground state calculated as two-valence-electron and four-valenceelectron system is given in TableVII. The final numbers, which are the average of two calculations are listed in the column "Final". Estimated uncertainties of all values are given in columns "Unc". Wavelengths for transitions to
TABLE IX: Absolute values of multipole reduced matrix elements obtained by CI+all-order two-valence-electrons (2-val) and four-valence-electrons (4-val) calculations in Sn-like $\mathrm{Pr}^{9+}$ ion (in a.u.).

\begin{tabular}{|c|c|c|c|c|}
\hline & \multicolumn{2}{|c|}{ Transition } & 2 -val & 4-val \\
\hline M1 & $5 p^{2}{ }^{3} P_{0}$ & $5 p^{2}{ }^{3} P_{1}$ & 1.273 & 1.274 \\
\hline M1 & $5 p 4 f^{3} F_{2}$ & $5 p^{2}{ }^{3} P_{1}$ & 0.323 & 0.359 \\
\hline M1 & $5 p 4 f^{3} G_{3}$ & $5 p 4 f^{3} F_{2}$ & 0.320 & 0.312 \\
\hline $\mathrm{E} 2$ & $5 p 2{ }^{3} P_{0}$ & $5 p 2{ }^{3} P_{2}$ & 1.901 & 1.864 \\
\hline $\mathrm{E} 2$ & $5 p^{2}{ }^{3} P_{0}$ & $5 p 4 f^{3} F_{2}$ & 0.232 & 0.110 \\
\hline $\mathrm{E} 2$ & $5 p^{2}{ }^{3} P_{1}$ & $5 p 4 f^{1} F_{3}$ & 0.623 & 0.577 \\
\hline $\mathrm{E} 2$ & $5 p 4 f^{3} G_{3}$ & $5 p^{2}{ }^{3} P_{1}$ & 0.183 & 0.170 \\
\hline $\mathrm{E} 2$ & $5 p 4 f^{3} F_{3}$ & $5 p^{2}{ }^{3} P_{1}$ & 1.133 & 1.071 \\
\hline $\mathrm{E} 2$ & $5 p 4 f^{3} G_{3}$ & $5 p 4 f^{3} F_{3}$ & 0.117 & 0.089 \\
\hline $\mathrm{E} 2$ & $5 p 4 f^{3} F_{2}$ & $5 p 4 f^{3} F_{4}$ & 0.662 & 0.686 \\
\hline $\mathrm{E} 2$ & $5 p 4 f^{3} G_{3}$ & $5 p 4 f^{1} F_{3}$ & 2.109 & 2.071 \\
\hline M3 & $5 p^{2}{ }^{3} P_{1}$ & $5 p^{2}{ }^{3} P_{2}$ & 13.369 & 14.162 \\
\hline M3 & $5 p^{2}{ }^{3} P_{0}$ & $5 p 4 f^{3} G_{3}$ & 0.427 & 0.328 \\
\hline M3 & $5 p^{2}{ }^{3} P_{0}$ & $5 p 4 f^{3} F_{3}$ & 4.906 & 5.246 \\
\hline
\end{tabular}

the ground state are given in last columns in $\mathrm{nm}$. The sensitivity coefficients $q$ for Sn-like $\mathrm{Pr}^{9+}$ and $\mathrm{Nd}^{10+}$ ions are given in Table VIII together with the corresponding CI+all-order transition energies and $K$ enhancement factors.

Comparison of multipole matrix elements obtained from CI+all-order 2-val and 4-val calculations in Sn-like $\mathrm{Pr}^{9+}$ ion is given in Table IX. The differences are few percent for most transitions, but significant for weak transitions, such as $E 25 p^{2}{ }^{3} P_{0}-5 p 4 f^{3} F_{2}$. It is expected since weak transitions are more sensitive to the admixtures of 
TABLE X: The CI+all-order multipole matrix elements $Z$ (in a.u.), transition rates $A_{r}$ (in $\mathrm{s}^{-1}$ ), and lifetimes $\tau$ (in sec) in Sn-like $\mathrm{Pr}^{9+}$ and $\mathrm{Nd}^{10+}$ ions. Transition energies $\Delta E\left(\right.$ in $\mathrm{cm}^{-1}$ ) and wavelengths $\lambda$ (in $\mathrm{nm}$ ) are obtained from final energy values given by Table VII The numbers in brackets represent powers of 10 .

\begin{tabular}{|c|c|c|c|c|c|c|c|c|c|}
\hline \multirow{2}{*}{$\begin{array}{c}\text { Ion } \\
\operatorname{Pr}^{9+}\end{array}$} & \multirow{2}{*}{$\begin{array}{r}\text { Term } \\
p 4 f^{3} G_{3}\end{array}$} & \multicolumn{2}{|c|}{ Transition } & \multicolumn{2}{|r|}{$\overline{\Delta E}$} & \multirow{2}{*}{ 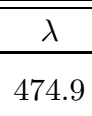 } & \multirow{2}{*}{$\begin{array}{c}Z \\
0.42712\end{array}$} & \multirow{2}{*}{$\begin{array}{c}A_{r} \\
2.001[-15]\end{array}$} & \multirow{2}{*}{$\begin{array}{c}\tau \\
4.997[+14]\end{array}$} \\
\hline & & $5 p^{2}{ }^{3} P_{0}$ & $5 p 4 f^{3} G_{3}$ & M3 & 21055 & & & & \\
\hline & $5 p 4 f^{3} F_{2}$ & $5 p^{2}{ }^{3} P_{0}$ & $5 p 4 f^{3} F_{2}$ & $\mathrm{E} 2$ & 23485 & 425.8 & 0.23230 & $8.635[-03]$ & 51.8 \\
\hline & & $5 p 4 f^{3} G_{3}$ & $5 p 4 f^{3} F_{2}$ & M1 & 2430 & 4115. & 0.31981 & $7.917[-03]$ & \\
\hline & $5 p 4 f^{3} F_{3}$ & $5 p 4 f^{3} F_{2}$ & $5 p 4 f^{3} F_{3}$ & M1 & 2697 & 3708 . & 1.67447 & $2.120[-01]$ & 4.718 \\
\hline & $5 p 4 f^{3} F_{4}$ & $5 p 4 f^{3} G_{3}$ & $5 p 4 f^{3} F_{4}$ & M1 & 7385 & 1354. & 1.89256 & $4.324[+00]$ & 0.227 \\
\hline & & $5 p 4 f^{3} F_{3}$ & $5 p 4 f^{3} F_{4}$ & M1 & 2258 & 4429. & 1.46389 & $7.394[-02]$ & \\
\hline & $5 p^{2}{ }^{3} P_{1}$ & $5 p^{2}{ }^{3} P_{0}$ & $5 p^{2}{ }^{3} P_{1}$ & M1 & 28474 & 351.2 & 1.27262 & $3.362[+02]$ & $2.975[-3]$ \\
\hline & $5 p^{2}{ }^{3} P_{2}$ & $5 p^{2}{ }^{3} P_{0}$ & $5 p^{2}{ }^{3} P_{2}$ & $\mathrm{E} 2$ & 35935 & 278.3 & 1.90140 & $4.852[+00]$ & 0.0838 \\
\hline & & $5 p 4 f^{3} G_{3}$ & $5 p^{2}{ }^{3} P_{2}$ & M1 & 14880 & 672.0 & 0.20488 & $7.461[-01]$ & \\
\hline & & $5 p 4 f^{3} F_{2}$ & $5 p^{2}{ }^{3} P_{2}$ & $\mathrm{E} 2$ & 12450 & 803.2 & 0.60441 & $2.448[-03]$ & \\
\hline & & $5 p 4 f^{3} F_{3}$ & $5 p^{2}{ }^{3} P_{2}$ & M1 & 9753 & 1025. & 0.90901 & $4.135[+00]$ & \\
\hline & & $5 p^{2}{ }^{3} P_{1}$ & $5 p^{2}{ }^{3} P_{2}$ & M1 & 7461 & 1340. & 0.99055 & $2.198[+00]$ & \\
\hline & $5 p 4 f{ }^{1} F_{3}$ & $5 p 4 f^{3} F_{2}$ & $5 p 4 f^{1} F_{3}$ & M1 & 30919 & 323.4 & 1.02292 & $1.192[+02]$ & $7.382[-3]$ \\
\hline & & $5 p 4 f^{3} G_{3}$ & $5 p 4 f^{1} F_{3}$ & $\mathrm{E} 2$ & 33349 & 299.9 & 2.10930 & $2.936[+00]$ & \\
\hline & & $5 p 4 f^{3} F_{4}$ & $5 p 4 f^{1} F_{3}$ & M1 & 25964 & 385.1 & 0.25958 & $4.545[+00]$ & \\
\hline & & $5 p^{2}{ }^{3} P_{2}$ & $5 p 4 f^{1} F_{3}$ & M1 & 18469 & 541.4 & 0.60127 & $8.776[+00]$ & \\
\hline \multirow[t]{15}{*}{$\mathrm{Nd}^{10+}$} & $5 p 4 f{ }^{5} G_{3}$ & $4 f^{23} H_{4}$ & $5 p 4 f^{5} G_{3}$ & M1 & 1062 & 9416 & 0.06522 & $1.963[-05]$ & $5.094[+04]$ \\
\hline & $4 f^{2}{ }^{3} H_{5}$ & $4 f^{2}{ }^{3} H_{4}$ & $4 f^{2}{ }^{3} H_{5}$ & M1 & 3059 & 3269 & 3.21593 & $7.259[-01]$ & 1.378 \\
\hline & $5 p 4 f^{1} D_{2}$ & $5 p 4 f^{5} G_{3}$ & $5 p 4 f^{1} D_{2}$ & M1 & 3488 & 2867 & 0.41514 & $3.945[-02]$ & 25.35 \\
\hline & & $4 f^{2}{ }^{3} H_{4}$ & $5 p 4 f{ }^{1} D_{2}$ & $\mathrm{E} 2$ & 4550 & 2198 & 0.66642 & $1.940[-05]$ & \\
\hline & $4 f^{23} H_{6}$ & $4 f^{2}{ }^{3} H_{5}$ & $4 f^{2}{ }^{3} H_{6}$ & M1 & 3162 & 3163 & 3.26836 & $7.007[-01]$ & 1.427 \\
\hline & $5 p 4 f^{3} F_{3}$ & $5 p 4 f^{1} D_{2}$ & $5 p 4 f^{3} F_{3}$ & M1 & 2188 & 4570 & 1.94864 & $1.533[-01]$ & 3.916 \\
\hline & & $5 p 4 f^{5} G_{3}$ & $5 p 4 f^{3} F_{3}$ & M1 & 5676 & 1762 & 0.36934 & $9.612[-02]$ & \\
\hline & & $4 f^{2}{ }^{3} H_{4}$ & $5 p 4 f^{3} F_{3}$ & M1 & 6738 & 1484 & 0.07115 & $5.967[-03]$ & \\
\hline & $4 f^{2}+5 p 4 f^{3} F_{2}$ & $5 p 4 f^{1} D_{2}$ & $4 f^{2}+5 p 4 f^{3} F_{2}$ & M1 & 3410 & 2933 & 0.29565 & $1.870[-02]$ & 43.92 \\
\hline & & $5 p 4 f^{5} G_{3}$ & $4 f^{2}+5 p 4 f^{3} F_{2}$ & M1 & 6898 & 1450 & 0.04406 & $3.437[-03]$ & \\
\hline & & $4 f^{2}{ }^{3} H_{4}$ & $4 f^{2}+5 p 4 f^{3} F_{2}$ & $\mathrm{E} 2$ & 7960 & 1256 & 0.93855 & $6.305[-04]$ & \\
\hline & $4 f^{2}+5 p 4 f{ }^{5} G_{4}$ & $5 p 4 f^{5} G_{3}$ & $4 f^{2}+5 p 4 f^{5} G_{4}$ & M1 & 6927 & 1444 & 1.57004 & $2.456[+00]$ & 0.365 \\
\hline & & $4 f^{23} H_{4}$ & $4 f^{2}+5 p 4 f^{5} G_{4}$ & M1 & 7989 & 1252 & 0.38552 & $2.271[-01]$ & \\
\hline & & $4 f^{23} H_{5}$ & $4 f^{2}+5 p 4 f^{5} G_{4}$ & M1 & 4930 & 2028 & 0.36808 & $4.865[-02]$ & \\
\hline & & $5 p 4 f^{3} F_{3}$ & $4 f^{2}+5 p 4 f^{5} G_{4}$ & M1 & 1251 & 7994 & 1.39879 & $1.148[-02]$ & \\
\hline
\end{tabular}

configurations which are omitted in the 2-val calculation.

The CI+all-order multipole matrix elements $Z$, transition rates $A_{r}$, and lifetimes $\tau$ in Sn-like $\mathrm{Pr}^{9+}$ and $\mathrm{Nd}^{10+}$ ions are presented in Table $\mathrm{X}$. Energies are final results presented in Table VII. The numbers in brackets represent powers of 10 . We highlight the case of $\operatorname{Pr}^{9+}$ where the lowest metastable state, $5 p 4 f^{3} G_{3}$, has a very long lifetime with $M 3495 \mathrm{~nm}$ transition to the ground state being in the optical range. Next two levels, $5 p 4 f^{3} F_{2}$ and $5 p 4 f{ }^{3} F_{3}$, also have optical transitions to the ground state and are metastable with $59 \mathrm{~s}$ and $5.3 \mathrm{~s}$ lifetimes. A relatively strong $M 1$ transition to the ground state from $5 p^{2}{ }^{3} P_{1}$ level at $351 \mathrm{~nm}$ may be potentially used for cooling and probing. Our $\mathrm{Nd}^{10+}$ transition property calculations assume that $4 f^{2} J=4$ level is the ground state. While several low levels of $\mathrm{Nd}^{10+}$ are long-lived, the corresponding transitions are all far in the infrared.

\section{CONCLUSION}

We carried out detailed high-precision study of Cd-like $\mathrm{Nd}^{12+}, \mathrm{Sm}^{14+}$ and $\mathrm{Sn}$-like $\mathrm{Pr}^{9+}, \mathrm{Nd}^{10+}$ atomic properties using a hybrid approach that combines configuration interaction and a linearized coupled-cluster method. These highly-charged ions are of interest for future experimental studies aimed at the development of ultra-precise atomic clocks and search for $\alpha$ variation. Energies, transition wavelengths, electric- and magnetic-multipole reduced matrix elements, lifetimes, and the sensitivity coefficients to $\alpha$ variation, $q$ and $K$, were calculated. Several methods to evaluate uncertainties of the results were developed. 


\section{Acknowledgement}

We thank C. W. Clark, C. Monroe, J. Tan, Yu. Ralchenko, J. R. Crespo López-Urrutia and P. Beiersdorfer for useful discussions. M.S.S. thanks School of Physics at the University of New South Wales, Sydney, Australia for hospitality and acknowledges support from Gordon Godfrey Fellowship, UNSW. This work was supported in part by US NSF Grant No. PHY-1212442. M.G.K. acknowledges support from RFBR Grant No. 14-02-00241. The work was partly supported by the Australian Research Council.
[1] C. W. Chou et al., Phys. Rev. Lett. 104, 070802 (2010).

[2] N. Hinkley et al., Science 341, 1215 (2013).

[3] B. J. Bloom et al., Nature, 506, 71 - 75 (2014).

[4] P. Dubé, A. A. Madej, Z. Zhou, and J. E. Bernard, Phys. Rev. A 87, 023806 (2013).

[5] Ichiro Ushijima, Masao Takamoto, Manoj Das, Takuya Ohkubo, and Hidetoshi Katori, arXiv:1405.4071 (2014).

[6] T. Rosenband, D. B. Hume, P. O. Schmidt, C. W. Chou, A. Brusch, L. Lorini, W. H. Oskay, R. E. Drullinger, T. M. Fortier, J. E. Stalnaker, et al., Science 319, 1808 (2008).

[7] E. Peik and C. Tamm, Europhys. Lett. 61, 181 (2003).

[8] J. C. Berengut, V. A. Dzuba, and V. V. Flambaum, Phys. Rev. Lett. 105, 120801 (2010).

[9] A. Derevianko, V. A. Dzuba, and V. V. Flambaum, Phys. Rev. Lett. 109, 180801 (2012).

[10] V. A. Dzuba, A. Derevianko, and V. V. Flambaum, Phys. Rev. A 86, 054502 (2012).

[11] J. K. Webb, J. A. King, M. T. Murphy, V. V. Flambaum, R. F. Carswell, and M. B. Bainbridge, Phys. Rev. Lett. 107, 191101 (2011).

[12] M. Hobein, A. Solders, M. Suhonen, Y. Liu, and R. Schuch, Phys. Rev. Lett. 106, 013002 (2011).

[13] Z. Andelkovic et al., Phys. Rev. A 87, 033423 (2013).

[14] M. Schwarz et al., Rev. Sci. Instrum. 83, 083115 (2012).

[15] O. O. Versolato et al., Hyperfine Interact. 214, 189 (2013).

[16] L. Gruber et al., Phys. Rev. Lett. 86, 636 (2001).

[17] S. M. Brewer, N. D. Guise, and J. N. Tan, Phys. Rev. A 88, 063403 (2013).

[18] M. S. Safronova, V. A. Dzuba, V. V. Flambaum, U. I. Safronova, S. G. Porsev, and M. G. Kozlov, Phys. Rev. Lett 113, 030801 (2014).

[19] M. S. Safronova, V. A. Dzuba, V. V. Flambaum, U. I. Safronova, S. G. Porsev, and M. G. Kozlov, submitted to Phys. Rev. A (2014).

[20] Yu. Ralchenko, F.-C. Jou, D. E. Kelleher, A. E. Kramida, A. Musgrove, J. Reader, W. L. Wiese, and K. Olsen, NIST Atomic Spectra Database (version 3.0.2). (National Institute of Standards and Technology, Gaithersburg, MD, 2005) available online at http://physics.nist.gov/asd3 [accessed 04 January 2006].
[21] A. N. Ryabtsev, S. S. Churilov, and Y. N. Joshi, Phys. Scr. 65, 227 (2002).

[22] Y. N. Joshi, A. N. Ryabtsev, and S. S. Churilov, J. Opt. Soc. Am. B 18, 1935 (2001).

[23] S. S. Churilov and Y. N. Joshi, Phys. Scr. 68, 128 (2003).

[24] S. S. Churilov, Y. N. Joshi, and A. N. Ryabtsev, Phys. Scr. 71, 43 (2005).

[25] M. G. Kozlov, Int. J. Quant. Chem. 100, 336 (2004).

[26] M. S. Safronova, M. G. Kozlov, W. R. Johnson, and D. Jiang, Phys. Rev. A 80, 012516 (2009).

[27] S. A. Kotochigova and I. I. Tupitsyn, J. Phys. B 20, 4759 (1987)

[28] V. A. Dzuba, V. V. Flambaum, and M. G. Kozlov, Phys. Rev. A 54, 3948 (1996).

[29] M. S. Safronova, M. G. Kozlov, and C. W. Clark, Phys. Rev. Lett. 107, 143006 (2011).

[30] S. G. Porsev, M. S. Safronova, and M. G. Kozlov, Phys. Rev. Lett. 108, 173001 (2012).

[31] M. S. Safronova, S. G. Porsev, M. G. Kozlov, and C. W. Clark, Phys. Rev. A 85, 052506 (2012).

[32] M. S. Safronova, M. G. Kozlov, and C. W. Clark, IEEE Transactions on Ultrasonics, Ferroelectrics, and Frequency Control 59, 439 (2012).

[33] M. S. Safronova, M. G. Kozlov, and U. I. Safronova, Phys. Rev. A 85, 012507 (2012).

[34] M. S. Safronova, S. G. Porsev, and C. W. Clark, Phys. Rev. Lett. 109, 230802 (2012).

[35] M. S. Safronova, U. I. Safronova, and S. G. Porsev, Phys. Rev. A 87, 032513 (2013).

[36] V. V. Dzuba, M. S. Safronova, and U. I. Safronova, Phys. Rev. A 90, 012504 (2014).

[37] V. V. Flambaum and J. S. Ginges, Phys. Rev. A 72, 052115 (2005).

[38] P. J. Mohr, B. N. Taylor, and D. B. Newell, The 2010 CODATA Recommended Values of the Fundamental Physical Constants (Web Version 6.0), database developed by J. Baker, M. Douma, and S. Kotochigova (National Institute of Standards and Technology Gaithersburg, MD, 2011), available online at http://physics.nist.gov/constants. 